\title{
Les mycobactéries d'origine animale isolées au centre Muraz de 1965 à 1968 Techniques d'isolement et d'identification. Résultats
}

\author{
por R. GIDEL, J. P. ALBERT, M. LEFÈVRE, M. MÉNARD ef M. RÉTIF \\ avec la collaboration rechnique de A. DJOKOUI
}

\begin{abstract}
RÉSUMÉ
Les auteurs soulignent tout d'abord l'importance des saisies pour tuberculose aux abattoirs de Bobo-Dioulasso. La fréquence de ces saisies a été de 9,61 p. 100 pour les bovins (82.961 anımaux inspectés) et de 2,15 p. 100 pour les porcins (16.992 animaux contrôlés) au cours des années 1965 d̀ 1968 incluse. Ils indiquent ensuite les jechniques qu'ils ont utilisées pour les prélèvements, l'isolement et l'identıfication des mycobactéries. Enfin, ils exposent les résultats obtenus concernant les exarnens directs, les cultures et l'identificatıon des mycobactéries isolées.

Parmi les 639 prétèvements reçus au Laboratoire, 298 cultures furent positives et 250 souches purent être identifiées.

Mycobocterium bovis est l'agent le plus fréquemment rencontré : 89,6 p. 100 des cas.

Mycobacterium tuberculosis a cependant été isolé dans 5,2 p. 100 des cas et des mycobactéries atypiques dans 5,2 p. 100 des cas également. Aucune souche de Nocardia farcınica n'a été isolée. Ces résultats montrent la rareté du farcin et l'imporfance de la tuberculose bovine dans les régions dont les animaux étaient originaires.

Les auteurs concluent en faisant part de leur intention d'entreprendre une étude bactériologique dans les régıons sahéliennes d'élevage où des enquêtes tuberculiniques récentes ont montré que des liens devaient exister entre tuberculoses humaine el bovine. Ces recherches permettront de préciser les types de mycobactéries en cause et de mieux connâ̂tre l'épidémiologie de la maladie dans ces régıons.
\end{abstract}

\section{1. - INTRODUCTION}

Depuis de nombreuses années, les vétérinaıres chargés de l'inspection des viandes à l'abattoir de Bobo-Dioulasso en Haute-Volto ont été frappés par la -fréquence des lésıons tuberculeuses chez les animaux abattus dans cet établissement. Dans certaines régions d'Afrique au contraire, la tuberculose semble exceptionnelle : c'est le cas du Tchad notamment. Par contre, dans ce pays et dans d'autres également, on rencontre, avec une fréquence relative, le farcın dû à 'Nocardia farcinica, dont les lésions peuvent évo- quer, pour l'inspecteur non averti, une tuberculose miliaire. En 1961, une étude sur le farcin a été effectuée aux abattoirs de Fort-Lamy au Tchad (PERPEZAT ef al., 1963). Sur 38.201 bovins abattus, 476 ganglıons suspects avaient été prélevés. Pour chacun d'eux, les auteurs ont isolé Nocardia forcinica, alors qu'aucun cas de fuberculose n'a été mis en évidence.

En ce qui concerne la Haute-Volta par contre, aucun cas de farcin n'a été signalé jusqu'à ce jour', ni à l'abattoir de Bobo-Dioulasso, ni à celui de Ovagadougou, ni dans ceux des centres secondaires. 
II nous a donc paru intéressant d'apporter la preuve bactériologique des nombreux cas de tuberculose dépistés macroscopiquement à l'abattolr de Bobo-Dioulasso et de précıser les types de mycobactéries en cause.

Les S/Sections Tuberculose et Zoonoses du Centre Muraz ont donc entrepris, à partir de 1965, l'identification des souches de myco-bactéries isolées à partir des prélèvements effectués sur les animaux abattus à Bobo-Dioulasso et présentanł des lésions tuberculeuses.

\section{2. - IMPORTANCE DES SAISIES POUR TUBERCULOSE A L'ABATTOIR DE BOBO-DIOULASSO}

Le pourcentage d'anımaux porteurs de lésions tuberculeuses varie considérablement d'une année d̀ l'autre. C'est aınsı que, chez les bovins, les taux suivants ont été observés au cours des 20 dernı̀res années :

$$
\begin{array}{r}
7,6 \text { p. } 100 \text { en } 1950 \\
19,5 \text { p. } 100 \text { en } 1953 \\
5,6 \text { p. } 100 \text { en } 1959 \\
14,8 \text { p. } 100 \text { en } 1962 .
\end{array}
$$

Ces variations s'expliquent par le fait qu'une part importante, de l'ordre de 65 à 75 p. 100, des bovins abattus sont d'origines très diverses, un certain nombre d'entre eux provenant des régions fortement infectées du Mali (Ségou, Macina, Mopti).

Au cours des années 1965 à 1968 incluse, 9.61 p. 100 des 82.961 bovins abattus, et 2,15 p. 100 des 16.992 porcins abattus étaient porteurs de lésions tuberculeuses (voir tableau 1). Pendant cette même pérıde, un certain nombre de cas de tuberculose ont également été observés chez les chevaux ( 9 cas sur 3.299 animaux inspectés), les moutons et les chèvres.

Comme nous l'avons sıgnalé ci-dessus, un pourcentage important des bovins abattus est d'origine étrangère. Par contre, les équins, ovins et caprins sont, pour la très grande majorité, d'origine voltaïque. Si on étudie la fréquence de la tuberculose bovine en fonction de l'origine des anımaux, on s'aperçoit que la maladie sévit avec une fréquence nettement plus élevée chez les anımaux d'origine étrangère. Ainsi, en 1968, sur 19.392 bovins abattus, 6.953 étaient d'origine voltaïque et 12.439 d'origine étrangère, la plupart du temps malienne. 1.929 cas de tuberculose ont été observés au total, dont 285 chez des bovins voltaiques, soit 4,10 p. 100 et $1.644 \mathrm{chez}$ des bovins étrangers, soit 13,22 p. 100 (chi $2=695$ pour deux degrés de liberté : la différence constatée est très hautement significative). Cette observation dolt attirer l'attention sur le danger que représente, pour le cheptel local, les animaux en transit provenant de régions contaminées. La réalité de ce danger nous est apparue également au cours de nos diverses enquêtes allergologiques (GIDEL et al., 1969).

\section{3. - TECHNIQUES UTILISÉES \\ POUR L'ISOLEMENT ET L'IDENTIFICATION DES MYCOBACTÉRIES}

\subsection{Récolte des prélèvements.}

Les prélèvements de fragments d'organes et de ganglions tuberculeux sont effectués, à l'abattoir de Bobo-Dioulasso, par les infirmiers spécıalıstes du Service de l'Elevage, chargés de l'inspection des viandes. Ces prélèvements sont placés aussitôt dans des boîtes de carton paraf-

\begin{tabular}{|c|c|c|c|c|c|}
\hline \multirow{2}{*}{$\begin{array}{l}\text { Espèce } \\
\text { animale }\end{array}$} & & $N \circ \mathrm{m} b \mathrm{re}$ & a $\mathrm{n} i \mathrm{ma} \mathrm{x}$ & & \multirow{2}{*}{$\begin{array}{l}\text { Pourcentage } \\
\text { d'animaux } \\
\text { tuberculeux à } \\
\text { l'abattage }\end{array}$} \\
\hline & $\begin{array}{l}\text { Abattus } \\
\text { et } \\
\text { inspectés }\end{array}$ & $\begin{array}{l}\text { Porteurs de } \\
\text { lésions } \\
\text { tuberculeuses }\end{array}$ & $\begin{array}{l}\text { Objets d'une } \\
\text { saisie totale pour } \\
\text { tuberculose }\end{array}$ & $\begin{array}{l}\text { Objets d'une } \\
\text { saisie totale } \\
\text { partielle pour } \\
\text { tuberculose }\end{array}$ & \\
\hline Bovins & 82.961 & 7.971 & 563 & 7.408 & 9,61 \\
\hline Porcins & 16.992 & 366 & 22 & 344 & $2, \quad 15$ \\
\hline Equins & 3.299 & 9 & 4 & 5 & 0,27 \\
\hline
\end{tabular}

TABLEAU $\mathbb{N}^{\circ}$ I

Nombre d'animaux abactus et inspectés à Bobo-Dioulasso au cours des années 1965 à 1968 incluses et résultats des saisies pour tuberculose. 
finé utılisées habıtueltement pour recueillir les crachats. Chaque prélèvement est accompagné d'une fiche d'identıfication numérotée (le même numéra étant reporté sur la boîte), indıquant l'espèce, la race, l'origine, le sexe, l'étendue et le type des lésions ainsi que les organes touchés et la nature de la sarsie (totale ou partielle). Ces prélèvements sont apportés au laboratoire de la Tuberculose du Centre Muraz. Les renseignements donnés par les fiches sont reportés sur un registre où figureront ultérieurement les résultats de l'examen direct, de la culture, de l'identification blochimique et, éventuellement, de l'antibiogramme.

\subsection{Traitement des prélèvements.}

\subsubsection{Homogénéisation au bromure de cétyl-} pyridinium (BERENCSI ef al., 1960).

Plusieurs parcelles des différents prélèvements provenant d'un même animal sont broyées au mortier stérıle en présence de sable et d'eau distillée stériles. Le produit du broyat est placé, après décantation, dans un tube à centrifugation à vis, stérile, et mis en contact avec du bromure de cétylpyridinium, dans la proportion de 1 volume de broyat pour 5 volumes d'une solution de bromure de cétyl-pyridinium à 5 p. 100 dans de l'eau distillée stérile. Le tube à centrifuger est ajors placé à l'étuve à 370 pendant 48 à $72 \mathrm{~h}$, ce temps étant fonction de l'état de conservation des prélèvements. Après ce délai, le tube est centrifugé à vitesse moyenne $(3.000 \mathrm{t} / \mathrm{mn})$ pendant 15 à $20 \mathrm{mn}$. Le surnageant est rejeté et le culot est difué dans 2 à $3 \mathrm{ml}$ d'eau distıllée stérile.

\subsubsection{Examen direct après homogénéisation.}

Une à deux gouttes du culot dilué sont étalées sur une lame porte-objet et séchées et fixées à l'alcool éthylıque flambé. Ce frottis est alors coloré selon la technique du Ziehl rapide à froid (LAPEYSSONNIE et CAUSSE, 1960) dont nous rappellerons succinctement les modalıtés.

Deux solutions sont utilisées pour cette coloration :

- Une solution A, dont la composition est la suivante:

Fuchsıne basique $\quad \ldots \ldots \ldots \ldots \ldots \quad 4 \mathrm{~g}$

Acide phénique .............. $12 \mathrm{~g}$

Alcool d̀ $95^{\circ} \quad \ldots \ldots \ldots \ldots \ldots \ldots .25 \mathrm{ml}$

Equ distillée q. s. ........... $300 \mathrm{ml}$
Elle se prépare en dissolvant la fuchsine dans l'acide phénique chauffé à $80^{\circ}$ au baln-marie. Après avoir laissé refroidir, et lorsque la solution est encore tiède, on ajoute l'alcool en agıtant. Puis on complète à $300 \mathrm{ml}$ avec de l'eau distillée et on filtre. Après filtration, on ajoute à cette solution 30 gouttes de Teepol (soit 10 gouttes pour $100 \mathrm{ml}$ environ) ou, à défaut, de Tween 80 . Cette solution dolt être filtrée chaque fois avant l'emploi.

- Une solution B, dont la composition est la suivante :

Alcool absolv $\quad . . \ldots \ldots \ldots .44$ parties

Acide sulfurique pur ........ 1 partie

Bleu de méthylène à 1 p. $100 \ldots 7$ parties

- Colorotion : le frottis est recouvert de solution A pendant $4 \mathrm{mn}$. Puis il est lavé à l'eau et recouvert de solution $B$ pendant $4 \mathrm{mn}$ également. Le frottis est ensuite lavé, séché, puis examiné à l'immersion. Cetie technique nous donne de bons résultats, tout en ayant l'avantage d'être plus rapide que la méthode classique à chaud.

\subsection{Mise en culture.}

Les milieux employés pour les cultures sont des milieux de LOEWENSTEIN-JENSEN à 0,75 p. 100 de glycérine, préparés au Laboratoire à partir de base déshydratée en sachets fournis par le Laboratoire BD-Mérieux. La préparation est effectuée selon la technique indiquée par ce Laboratoire. Les œufs utılisés sont des œufs du jour provenant de volailles sélectionnées et contrôlées sanitairement par le Service de l'Elevage. Une fois préparé, le milieu est réparti stérilement, sous lampe à ultraviolet, dans des tubes de Coletsos à encoche, à raison de $8 \mathrm{ml}$ par tube. Ces tubes sont ensuite placés en position horizontale au bain-marie coagulateur. $1 / 5$ sont enfin placés à l'étuve à $37^{\circ}$ pendant 48 h pour contrôle de stérılıté, puis conservés au réfrigérateur à +40 jusqu'à leur utilisation qui doit se faire dans les 15 jours.

Le culot dilué est ensemencé en nappes sur 2 tubes de LOEWENSTEIN-JENSEN. Ceux-ci sont portés, non capuchonnés, à l'étuve à 370 pendant $24 \mathrm{~h}$, puls capuchonnés et remis à l'étuve. Les tubes en culture sont surveillés régulièrement, de façon à noter l'apparition des premières colonies et à élıminer éventuellement les tubes souillés. Au bout de 4 mois, les milieux où 
aucune culture n'apparaît sont contrôlés au Ziehi et, si celui-ci est négatif, ils sont élimınés. Les tubes positifs sont conservés jusqu'à ce que la culture soit suffisante pour effectuer les tests biochımiques. L'aspect des colonies (lisses ou rugueuses; isolées ou en nappes; présence ou absence de pigmentation) est noté sur le registre. Le bacille bovin poussant mal sur LOEWENSTEINJENSEN au sortir de l'animal et les cultures étant lentes à obtenir, nous allons dorénavant ensemencer, en même temps que le milieu de LOEWENSTEIN-JENSEN à 0,75 p. 100 de glycérine, un tube de LOEWENSTEIN sans glycérine et un milieu au pyruvate de soude à 0,50 p. 100 (STONEBRINK, 1952).

\subsection{Photo-induction.}

Cette recherche est effectuée en ensemençant 3 tubes de LOEWENSTEIN-JENSEN, dont 2 sont enveloppés de papier noir. Lorsque le tube découvert montre une culture en pleine croissance, on développe l'un des 2 tubes et on l'expose 30 à $60 \mathrm{mn}$ à $50 \mathrm{~cm}$ environ d'une lampe de $40 \mathrm{~W}$. Puis on le recouvre et on le remet à l'étuve. Le lendemain, on découvre les 2 tubes et on compare l'aspect des colonies. Si les colonies qui ont été exposées à la lumière présentent une pigmentation jaune-orange par rapport à celles du tube témoin dont les colonies sont restées blanches, la souche est dite photochromogène. En ce qui concerne les mycobactéries dites scotochromogènes, c'est-à-dire d'emblée pigmentées en jaune ou orange pendant le séjour à l'étuve à l'obscurité, la photo-induction renforce la pigmentation.

\subsection{Tests biochimiques.}

Nous décrivons succinctement les différents tests utilisés pour l'identification de nos souches de mycobactéries.

\subsubsection{Recherche de l'acide nicotinique ou «Niacin test».}

La recherche de la production d'acide nicotinique est un test précieux car il permet à lui seul d'identifier $M$. tuberculosis qui est le seul, avec $M$. microti (bacille de WELLS) et certaines variétés de $M$. borstelense à donner une réaction positive. Toutefols, le type ofricanum de $M$. tuberculosis découvert récemment (CASTELS et al.,
1968 ef 1969) donne un niacin-test variable selon les souches.

Ce test est effectué sous hotte ou devant une fenêtre ouverte du fait de l'utilısation de bromure de cyanogène qui sert à révéler la présence d'acide nicotinique. Cette recherche se fait sur des cultures en milieu de LOEWENSTEIN-JENSEN abondantes et en pleine croissance. Elle consiste à déposer sur le milieu, à l'aide d'une pipette graduée, $0,5 \mathrm{ml}$ d'une solution d'aniline à 4 p. 100 dans l'alcool à 95 p. 100. Les tubes sont ensuite placés en position horizontale pendant $10 \mathrm{mn}$ environ. Puis on ajoute, avec une seringue munie d'une longue aiguille, $0,5 \mathrm{ml}$ d'une solution à 10 p. 100 de bromure de cyanogène dans l'eau distillée. La lecture s'effectue immédiatement, car la pigmentation s'atténue ensuite. La présence d'acide nicotinique se traduit par une coloration jaune des colonies et une coloration jaune à vert-jaune du liquide. Lorsque la réaction est négative, les colonies ne se colorent pas ef le liquide est de couleur verte. Enfin la réaction est dite douteuse si les colonies ne sont pas pigmentées en jaune mais si le liquide est vertjaune.

\subsubsection{Recherche conjointe de la catalase et de} la peroxydase à $22^{\circ}$ ef $70^{\circ}$ (CANETTI ef al. 1968).

La recherche de ces deux enzymes est faite sur des cultures jeunes. La présence de la catalase dans une souche donnée se traduit par un dégagement gazeux lorsque l'on met cette souche en présence d'eau oxygénée, tandis que la présence de la peroxydase se traduit par un noircissement des colonies lorsque celles-ci sont mises en présence d'equ oxygénée et d'un substrat oxydable tel que catéchol ou benzidine. La technique est la suivante : dans 2 tubes à hémolyse contenant 2 gouttes d'eau distillée, on introduit une anse de culture, soit environ $10 \mathrm{mg}$. L'un des 2 tubes est porté au bainmarie à $70^{\circ}$ pendant $15 \mathrm{mn}$ et refroidı. On introduit alors dans les 2 tubes, $1 \mathrm{ml}$ de la solution suivante, qui se conserve 2 semaines au réfrigérateur (solution de Bogen).

Pyrocatéchine ............. $0,1 \mathrm{~g}$

Eau oxygénée à 110 volumes .. $\quad 0,5 \mathrm{ml}$

Tween $80 \ldots \ldots \ldots \ldots \ldots \ldots . \ldots \ldots$ 1,25 ml

Eau distıllée.............. $100 \mathrm{ml}$

Une première lecture s'effectue au bout 
d'une heure pour la catalase ef la peroxydase ef une deuxième lecture après $18 h$, pour contrôle de la peroxydase, certaines souches n'étant peroxydase positives que tardivement. La formation d'une mousse plus ou moins abondante indique la présence d'une catalase, tandis que la peroxydase est décelée par la couleur du culot, une teinte brun-rouge signifiant la présence de peroxydase. Cependant, l'appréciation des teintes est parfols délicate.

Toutes les mycobactéries, à l'exception des souches de $M$. tuberculosis et $M$. bovis isoniazidorésistantes sont catalase positives. Les mycobactéries sont en général peroxydase négatives, à l'exception des souches de $M$. tuberculosis et $M$. bovis isoniazido-sensibles.

\subsubsection{Réduction des nitrates (VIRTANEN, 1960).}

Certaines mycobactéries possédant une nitrate réductase réduisent les nitrates en nitrites. La présence de nitrite de sodium pourra être décelée par la réaction de GRIESS.

Cetfe recherche se fait sur des cultures jeunes, en pleine croissance, âgées de molns d'un mois. Dans un tube à hémolyse confenant deux gouttes d'eau distillée, on introduit $10 \mathrm{mg}$ environ de bacilles (une anse pleine). On ajoute $2 \mathrm{ml}$ d'une solution de nitrate de soude à 0,085 p. 100 en tampon phosphate $\left(\mathrm{NO}_{3} \mathrm{Na}: 0,085 \mathrm{~g} ; \mathrm{PO}_{4} \mathrm{H}_{2} \mathrm{~K}\right.$ : $0,117 \mathrm{~g}: \mathrm{PO}_{4} \mathrm{HNa}_{2}-12 \mathrm{H}_{2} \mathrm{O}: 0,485 \mathrm{~g}$; eau distillée : $10 \mathrm{ml}$ ). On porte 2 heures à l'étuve à 370 . On ajoute successivement : deux gouttes du réactif $\mathrm{A}$ (acide sulfanilique : $0,8 \mathrm{~g}$; acide acétique : $30 \mathrm{ml}$; eau distıllée : $100 \mathrm{ml}$ ) et deux gouttes du réactif $B$ ( $\alpha$-naphtylamıne : $0,5 \mathrm{~g}$; acide acétique : $30 \mathrm{ml}$; eau distillée : $100 \mathrm{ml}$ ). La présence de nitrites se traduit par l'apparition immédiate d'une coloration rouge-violette.

Les souches de $M$. fuberculosis, qu'elles soient sensibles ou résistantes à l'isoniazide, rédusent les nitrates en nitrites, à l'exception des souches de type ofricanum pour lesquelles cette opération est en général négative. La plupart des souches de $M$. bovis ne réduisent pas les nitrates. Quant aux mycobactéries dites atypiques, elles se comportent de façon variable selon les espèces.

\subsubsection{Recherche des activités amidosiques (BÖNICKE, 1958).}

Cette recherche est indiquée pour l'identifi- cation des mycobactéries dites atypiques. Elle doif être faite sur des cultures en pleine croissance car il faut une quantité importante de germes. Cinq amides ont été utilisés aux concentrations suivantes :

pour $100 \mathrm{ml}$ d'eau distitlée :

$\begin{array}{lr}\text { Urée } \ldots \ldots \ldots \ldots \ldots \ldots \ldots \ldots & 9,84 \mathrm{mg} \\ \text { Nicotinamide } \ldots \ldots \ldots \ldots \ldots & 20,00 \mathrm{mg} \\ \text { Acétamide } \ldots \ldots \ldots \ldots \ldots \ldots & 9,68 \mathrm{mg} \\ \text { Benzamide } \ldots \ldots \ldots \ldots \ldots \ldots & 19,85 \mathrm{mg} \\ \text { Succinamıde } \ldots \ldots \ldots \ldots \ldots \ldots & 19,00 \mathrm{mg}\end{array}$

Ces solutions sont stérilisées par chauffage à l'autoclave à $100^{\circ}$ pendant $30 \mathrm{mn}$. Elles peuvent être conservées 8 jours au réfrigérateur.

La formation d'ammonıaque est décelée par la technique de RUSSEL qui utilise 3 réactifs :

- Une solution de sulfate de manganèse à $0,003 \mathrm{M}$ obtenue en dissolvant $66,9 \mathrm{mg}$ de $\mathrm{SO}_{4} \mathrm{Mn}$, $4 \mathrm{H}_{2} \mathrm{O}$ dans $100 \mathrm{ml}$ d'eau distillée :

- Un réactif phénolique préparé comme suit : $25 \mathrm{~g}$ d'acide phénıque sont dissous dans $10 \mathrm{ml}$ d'eau distillée. Après agitation vigoureuse, on ajoute $54 \mathrm{ml}$ de $\mathrm{NaOH}, 5 \mathrm{~N}$; on remue jusqu'à dissolution totale, puis on complète à $100 \mathrm{ml}$ avec de l'eau distillée et on conserve cette solution en flacons teintés bruns ;

- Une solution d'hypochlorite de calcium préparée de la façon suivante : dissoudre $25 \mathrm{~g}$ de $\mathrm{Cl}_{2} \mathrm{CaO}$ (hypochlorite de calcium) dans $300 \mathrm{ml}$ d'eau distillée chaude. Ajouter, en agitant, $135 \mathrm{ml}$ d'une solution à 20 p. 100 de carbonate de potassium. Faire bouillir quelques minutes pour élıminer l'ammoniaque. On vérifie, par addition d'oxalate de potassium ou d'acide oxalique à 1 p. 100, qu'il n'existe plus d'ions $\mathrm{Ca}$ dans la solution. Sinon, on ajoute du carbonate de potassium pour éliminer l'excès de calcium. Puis on complète jusqu'à $500 \mathrm{ml}$ avec de l'eau distillée et on filtre. Cette solution est également conservée en flacons teintés bruns au réfrigérateur.

- Technique de la réoction : Les colonies d'une culture abondante sur milieux de LOEWENSTEIN-JENSEN sont prélèvées et mises en suspension dans de l'eau physiologique stérile de manlère à avoir une concentration en germes d'au moins $10 \mathrm{mg} / \mathrm{ml}$. Pour chaque amide, on utillse $1 / 2 \mathrm{ml}$ de suspension bactérienne. II faut donc prévoir une quantité suffisante de cette 
suspension pour pratiquer le test sur les différentes amides. Après avoir procédé à une homogénéisation complète, on effectue deux lavages successifs des bactérıes avec de l'eau physiologique stérile. Après la deuxième centrifugation, le culot est ensuite repris en tampon phosphate $\mathrm{M} / 15$ d̀ $\mathrm{pH} 7,2,\left(\mathrm{PO}_{4} \mathrm{HNa}_{2}, 12 \mathrm{H}_{2} \mathrm{O}=1,718 \mathrm{~g}\right.$; $\mathrm{PO}_{4} \mathrm{H}_{2} \mathrm{~K}=0,254 \mathrm{~g}$; eau distillée : $100 \mathrm{ml}$ ), de façon à obtenır le même volume que celuı de la suspension précédente. On répartit ensulte $1 / 2 \mathrm{ml}$ de cette suspension dans des tubes à hémolyse contenant $1 / 2 \mathrm{ml}$ de la solution d'amide $0,00164 M$ (vorr ci-dessus la préparation des solutions des différentes amides). Puis les tubes sont placés à l'étuve d̀ 370 pendant 4 à 12 -18 h (4 heures pour les mycobactéries à crolssance rapide; 12 à $18 \mathrm{~h}$ pour les mycobactéries à croissance lente).

Après ce délai, la présence d'ammoniaque est décelée par la technique de RUSSEL qui consiste à ajouter à chaque fube : nèse,

- $0.1 \mathrm{ml}$ de la solution de sulfate de manga-

- $1 \mathrm{ml}$ de réactif phénolique,

$-0,5 \mathrm{ml}$ de la solution d'hyposulfite.

Après agitation, les tubes sont placés durant 15 à $20 \mathrm{mn}$ au bain-marie à 1000 . La présence d'ammoniaque se traduit par une coloration bleu-vert.

\subsection{Sensibilité aux antibiotiques (CANETTI et al. 1963 et 1968).}

La technique utilisée est la méthode des proportions de CANETTI, RIST et GROSSET (1963). Lo sensibilité est recherchée vis-à-vis des 3 antibıotlques majeurs : isoniazide (l. N. H.), acide paraamino-salycilique (P. A. S.), streptomycine.

Les deux concentrations suivantes sont utilisées pour chaque antibiotique :

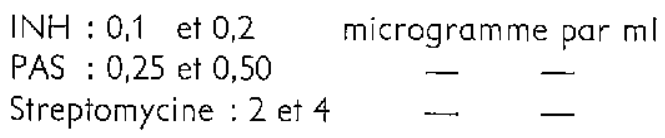

Ces solutions d'antibiotiques sont incorporées aux milieux de LOEWENSTEIN-JENSEN avant coagulation.

Les dilutions bacillaires à ensemencer sont les dilutions $10^{-3}$ ef $10^{-5}$ microgrammes par $\mathrm{m}$ !

Aux concentrations précédemment indiquées, les proportions critıques au-delà desquelles la souche est considérée comme résistante sont les suivantes:

$$
\begin{array}{ll}
\text { INH } \ldots \ldots \ldots \ldots . & 10 \text { p. } 100 \text { ef } 1 \text { p. } 100 \\
\text { PAS } \ldots \ldots \ldots \ldots . & 10 \text { p. } 100 \text { et } 1 \text { p. } 100
\end{array}
$$

Streptomycine .. 50-100 p. 100 ef 1 p. 100

\subsection{Test́ au Pyrazinamide.}

Nous n'avons pas pratiqué ce test jusqu'à présent. Toutefois, vu son intérêt pour le dépistage des souches de type africanum qui sont très sensibles à cet antibiotique, nous envisageons désormals sa mise en cuvre selon la technique de RIST (CANETTI et GROSSET, 1968). Pour pallier à la principale difficulté de ce test, à savoir la croissance très lente des bacilles tuberculeux sur le milıu utilisé, du fait d'un $\mathrm{pH}$ de 4,9 do à la présence du pyrazınamide, CASTETS $(M)$, RIST (N) et BOISVERT $(H)(1969)$, recommandent de déposer sur le milieu, avant l'ensemencement, $0,2 \mathrm{ml}$ de pyruvate de soude à 15 p. 100.

En présence de 50 microgrammes de pyrazinamide par millititre de milieu de LOEWENSTEINJENSEN à pH 4,9, la croissance du type africaın est inférieure d 1 p. 100 de la population ensemencée.

\subsection{Test au TCH.}

La recherche de la sensibilité vIs-à-vis de I'hydrazide de l'acide thiofène-2-carboxylique $(\mathrm{TCH})$ est partıculièrement intéressante pour l'identification des souches de $M$. bovis et du type africain de $M$. tuberculosis. En effet, ces souches sont sensibles au TCH, alors que celles de $M$. tuberculosıs (type humaın) y sont résistantes. Sur les conseils du docteur N. RIST, nous allons dorénavant effectuer ce test systématiquement suivant la technıque utilisée pour les antibiogrammes. Les concentrations utilisées sont de 2 et 5 microgrammes de TCH par millilitre de milieu et la proportion critıque au-delà de laquelle la souche est considérée comme résıstante est 1 p. 100.

La résistance à l'INH étant crolsée avec celle au TCH, le comportement à l'égard du $\mathrm{TCH}$ ne permet donc pas de différencier les souches bovines et afrıcaınes isoniazido-résistantes des souches humaines. La recherche de la sensibilité à I'INH doit donc toujours être faite lorsqu'on effectue le test au TCH afin de pouvoir interpréter correctement celui-ci. 


\section{4. - RÉSULTATS}

639 prélèvements ont été adressés au Laboratoire au cours des années 1965, 1966, 1967 et 1968 (voir tableau 2). Ces prélèvements concernaient des bovıns (zébus, 507 fois ; taurıns, 77 fols) ; des porcins ( 27 fois) ; des équins ( 8 fois) ; divers ovins (10 fois); des caprins ( 7 fois) ; divers animaux (poules, 2 fois; canards, 1 fois ; chiens. 2 fois). Ces prélèvements provenaient pour 221 d'entre eux de saisies totales et pour 413 d'entre eux de saisies partielles; dans 5 cas (poules, canards, et chiens), il s'agissait de prélèvements effectués par les agents du Service de l'Elevage à l'occasion d'autopsies.

TABLEAU $\mathrm{H}^{\circ}$ II

Nombre de prélèvements tuberculeux adressés au Iaborazoire au cours des années 1965 à 1968 incluses pour chaque espèce animale avec indications des saisies pratiquées.

\begin{tabular}{|c|c|c|c|c|}
\hline & \multirow{2}{*}{ 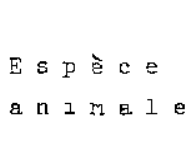 } & \multirow{2}{*}{$\begin{array}{l}\text { Mombre de } \\
\text { prélèvements } \\
\text { tuberculeux } \\
\text { auressés au } \\
\text { Laboratoire }\end{array}$} & \multicolumn{2}{|c|}{$\begin{array}{l}\text { Nature des sarsies pratiquées } \\
\text { aux abattoirs sur les animaux } \\
\text { objets des préièvements }\end{array}$} \\
\hline & & & Totales & ?artielles \\
\hline \multirow{2}{*}{ 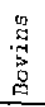 } & Eềus & 507 & 168 & 339 \\
\hline & Taurins & 77 & 20 & 48 \\
\hline \multicolumn{2}{|r|}{ Porcins } & 27 & 13 & IE \\
\hline \multicolumn{2}{|r|}{ Equins } & 8 & 3 & 5 \\
\hline \multicolumn{2}{|r|}{ Ovins } & 10 & 6 & it \\
\hline \multicolumn{2}{|r|}{ Caprins } & 5 & 4 & 1 \\
\hline \multicolumn{2}{|r|}{ Poules (I) } & 2 & 2 & - \\
\hline \multicolumn{2}{|r|}{ Canerd (1) } & ] & 1 & - \\
\hline \multicolumn{2}{|r|}{ Chiens (1) } & 2 & 2 & - \\
\hline \multicolumn{2}{|r|}{ Total } & 639 & 226 & 413 \\
\hline
\end{tabular}

$(1)=$ Il s'Aglt de prélèrementa effectués par les agents du Service de l'filevage è l'occasion d'autopsies.

\subsection{Résultats des examens directs. (voir tableau 3).}

Au début de l'enquête, les examens directs n'ont pas été pratıqués de façon systématique.
C'est la raison pour laquelle ces résultats n'ont pu être donnés pour 116 prélèvements. En ce qui concerne les 523 autres, ces examens ont été positifs dans 160 cas (30,5 p. 100) et négatifs dans $363 \operatorname{cas}(69,5$ p. 100).

\section{TABLEAU NO III}

Pésultats des exarens airects et des cultures effectués à partir des prélèvements reçus.

\begin{tabular}{|c|c|c|c|c|c|c|c|c|}
\hline \multicolumn{3}{|c|}{160 positifs } & \multicolumn{3}{|c|}{363 négatifs } & \multicolumn{3}{|c|}{116 non effectués } \\
\hline \multicolumn{3}{|c|}{ Cultures } & \multicolumn{3}{|c|}{ Cultures } & \multicolumn{3}{|c|}{ Cultures } \\
\hline Positives & Négatives & Eliminées & Positives & Négetives & Eliminées & Positives & Négatives & Eliminées \\
\hline 90 & 35 & 35 & 156 & 104 & 103 & $5 ?$ & 47 & 17 \\
\hline
\end{tabular}


4.2. Résultats des cultures. (voir tableau 3).

Sur les 639 prélèvements mis en cultures, 298 ont donné des cultures positives et 186 des cultures négatives. 155 cultures en cours ont dô être éliminées, soit par suite de souillures, soit pour la plupart d'entre elles, à la suite d'un accident d'étuve. 51 on ne tient pas compte de ces cultures éliminées, on obtıent donc 46,6 p. 100 de résultats positifs pour les prélèvements mis en cultures.

Les 160 examens directs positifs ont donné, du point de vue cultures : 90 cultures positives, 35 négatıves et 35 élıminées. En ne tenant pas compte de ces dernières, on obtient donc 72 p. 100 de cultures positives et 28 p. 100 de cultures négatives. L'existence ce ces 28 p. 100 de bacilles visibles et non isolés en cultures peut être imputée notamment à deux facteurs. D'une part, Il est bien certain que le milieu de LOEWENSTEINJENSEN à 0,75 p. 100 de glycérine n'est pas très favorable au développement des Mycobacterium bovis. II est donc probable que certaines souches de ce type n'ont pu se développer et leur existence peut expliquer une partie de ces échecs. Mais d'autre part, l'étude des résultats en ce qui concerne les isolements de mycobactéries d'origine humaine a montré que le même phénomène, quoique à un degré moindre, pouvait y être observé également (15 p. 100 de bacilles visibies et non isolés en cultures au cours de la période 1966-1968).
Une étude plus attentive de la distribution de ce phénomène a alors montré qu'il apparalssait ovec une fréquence plus grande pendant de courtes périodes correspondant à l'utilisation des fins de lots de milieux de culture. L'emploi de milieux frais, préparés depuis morns de 15 jours, devait entraîner une nette amélioration. Toutefois, malgré ces précautions, certains bacilles visibles ne poussent pas en cultures. Le pourcentage de ces bacilles est de l'ordre de 10 à 12 p. 100. II s'agit vraisemblablement de bacilles isoniazidorésistants qui poussent mal en milieu de LOEWENSTEIN-JENSEN.

\subsection{Résultats des identifications. (voir tableau 4).}

\subsubsection{Critères retenus pour les identifications.}

Ces identifications sont prononcées après étude des caractères culturaux et biochımiques.

Pour Mycobacterium tuberculosis, les colonies sont rugueuses et eugoniques, apparaissant entre 10 à 30 jours. La catalase et la peroxydase sont en général positives à $22^{\circ}$ et négatives à $70^{\circ}$. Le niacin test et les nitrates sont positifs ainsi que la recherche de la nicotinamidase. Enfin, les souches de ce type sont résistantes au $\mathrm{TCH}$ et très sensibles au pyrazinamide.

Pour Mycobacterlum bovis, les colonies sont lisses, bombées, luisantes ef dysgoniques, apparaissant très lentement sur milieux de LOEWENSTEIN-JENSEN (souvent après 2 mois; parfois même après 3 mois). Catalase et peroxydase

TABLEAU NO ${ }^{\circ}$ IV

Fésultats ces iäentifications des mycobactêries źsolées pour chaque espèce animele.

\begin{tabular}{|c|c|c|c|c|c|c|c|c|c|c|c|c|c|}
\hline \multirow{3}{*}{$\begin{array}{l}\text { Souches } \\
\text { Espece } \\
\text { guimele }\end{array}$} & \multirow{2}{*}{\multicolumn{3}{|c|}{$\frac{\text { Y. tuberculosis }}{\text { I.N.A. }}$}} & \multicolumn{3}{|c|}{ M. bcvis } & \multicolumn{6}{|c|}{ M. $\quad \varepsilon t y p i q u \in s$} & \multirow{3}{*}{$\mathrm{T}$} \\
\hline & & & & & $I, N$ & $\overline{1}$ & \multirow{2}{*}{$\begin{array}{l}\text { IM. } \\
\text { aquae } \\
I I\end{array}$} & \multirow{2}{*}{$\begin{array}{l}\text { M. } \\
\text { aquae } \\
\text { III }\end{array}$} & \multirow{2}{*}{ batteyi } & \multirow{2}{*}{$\begin{array}{c}\text { Y. } \\
\text { borstelense }\end{array}$} & \multirow{2}{*}{ Mansasi } & \multirow{2}{*}{ non } & \\
\hline & $S$ & $R$ & $\begin{array}{c}\text { non } \\
\text { précisé }\end{array}$ & 5 & $\mathrm{R}$ & $\begin{array}{c}\text { non } \\
\text { précisé }\end{array}$ & & & & & & & \\
\hline 孚 Zébus & & & 7 & 10 & 17 & 165 & 1 & 1 & 1 & 1 & 1 & 5 & 209 \\
\hline Taurins & & & 3 & & 2 & 18 & & & & & & 1 & 24 \\
\hline Porcins & 1 & & 2 & 1 & & 6 & & & 1 & & & 2 & 12 \\
\hline Ovins & & & & & & 3 & & & & & & & 3 \\
\hline Equins & & & & 1 & & 1 & & & & & & & 2 \\
\hline Total & 1 & & 12 & 12 & 19 & 193 & 1 & 1 & 2 & 1 & 1 & 7 & 250 \\
\hline
\end{tabular}


présentent les mêmes caractères que pour le bacılle humain, c'est-à-dire qu'elles sont positives à $22^{\circ}$ et négatives à $70^{\circ}$. Niacin test, nitrates et nicotinamidase sont en général négatifs. Enfin, $M$. bovis est sensible au TCH et résistant au pyrazinamide.

En ce qui concerne les mycobactéries dites atypiques, l'étude des amidases est nécessaire pour parvenir à l'identificatıon complète. Malgré tout, celle-ci n'est pas toujours aisée et nécessite parfois l'envol de la souche à un laboratoire spécialisé.

La classification de RUNYON permet de distinguer les groupes suivants :

- Groupe I : Mycobactéries photochromogènes, c'est-à-dire mycobactérıes dont les colonies non pigmentées à l'obscurité se pigmentent après exposition à la lumière ;

- Groupe II : Mycobactéries scotochromogènes, c'est-à-dıre mycobactéries à croissance lente dont les colonies se pıgmentent à l'obscurité :

- Groupe III : Mycobactéries non chromogènes, c'est-à-dire mycobactéries dont les colonies à croissance lente ne se pigmentent habituellement ni à la lumière nı à l'obscurité ;

- Groupe IV : Mycobactéries à croissance rapide dont les colonies se développent en 3 à 4 jours.

Enfin, dans les résultats qui vont suivre, nous ne faisons pas mention des bacilles tuberculeux de type africain. En effet, nous ne pratiquions pas, jusqu'à présent, les deux tests les plus caractéristiques de ce type, à savoır, la recherche de la sensibilité au TCH et au pyrazinamide, Les colonies sont dysgoniques, mais leur croissance est en général plus rapide que celle de $M$. bovis. Elle n'excède pas 4 à 6 semaines. Le test de la niacine est variable et la réduction des nitrates habituellement négatıve. Ce dernier caractère est toutefors sujet aussi à variations.

II n'est donc pas impossible que quelques souches étiquetées $M$. bovis appartiennent en réalité au type afrıcaın. Cette hypothèse pourra être confirmée ou infirmée rétrospectıvement. En effet, la recherche minutieuse des souches d'origine animale appartenant à ce type sera désormaıs effectuée systématıquement et les souches dépistées ou suspectes envoyées à l'Institut Pasteur de Paris pour confirmation.

Parmi les 298 cultures positives, 250 souches au total furent identifiées. Les autres n'ont pu l'être de façon précıse pour des raisons diverses (difficultés de la culture au repiquage et surtout perte d'un lot de souches repiquées par suite d'un accident d'étuve). Ces souches ont donc été éliminées.

\subsubsection{Résultats globaux des identifications.}

- Mycobacterium tuberculosis a été isolé 13 fois sur 250 , soit dans 5.2 p. 100 des cas. Dans 10 cas les prélèvements provenaient de bovins ef dans 3 cas de porcins.

- Mycobacterium bovis a été isolé 224 fois sur 250 , salt dans 89,6 p. 100 des cas. Dans 212 cas les prélèvements provenalent de bovins (zébus : 192 fois ; taurins : 20 fols) ; dans 7 cas de porcins ; dans 3 cas d'ovıns et dans 2 cas d'équins.

- Des mycobactéries atypıques ont été isolées 13 fois sur 250 , soit dans 5,2 p. 100 des cas.

Les souches isolées se répartissent ainsi selon la classification de RUNYON :

Groupe I : $M$. konsosii : 1 fois chez un bovin zébu ;

Groupe II : $M$. aquae $\|: 1$ fois chez un bovin zébu ;

M. aquae $I I I: 1$ fois chez un bovin zébu également :

Groupe ill : $M$. batfeyi : 2 fois dont l'une chez un bovin zébu ef l'autre chez un porcin :

Groupe IV : $M$. borstelense : 1 fois chez un bovin zébu.

7 Mycobactéries n'ont pu être identıfiées de façon exacte par manque de concordance entre les différents test pratiqués ( 5 fois chez des bovins zébus: 1 fois chez un bovin taurin; 1 fols chez un porcin).

\subsubsection{Résultats des identifications par espèce animale.}

- Bovins : 233 souches au total ont été isolées, dont 209 en provenance de zébus et 24 en provenance de taurins.

Parmi les 233 souches obtenues le bacille humain a été ısolé 10 fois soit dans 4,3 p. 100 des cas, le bacille bovin 212 fois, soit dans 91 p. 100 des cas ef les mycobactéries atypiques 11 fois, soit dans 4,7 p. 100 des cas.

- Porcins : 12 souches au total ont été isolées. 3 fois, il s'agissait de $M$. tuberculosis ; 7 fois de $M$. bovis et 2 fols de mycobactéries atypiques. 
- Ovins : 3 souches ont été isolées et chaque fois, il s'agissait de M. bovis.

- Equins : 2 souches ont été isolées, et dans les 2 cas, il s'agissalt de $M$. bovis.

\subsubsection{Etude des lésions provoquées par $M$. tuber- culosis et les mycobactéries difes afypiques.}

Dans leur ensemble, ces lésions ne diffèrent pas de celles observées avec $M$. bovis.
En ce qui concerne $M$. tuberculosis les divers types de lésions rencontrées chez chaque espèce animale figurent dans le tableau 5. On peut constater que, contrairement à ce qui est observé en France où ce type de bacille n'engendre chez les bovins qu'une tuberculose fermée et spontanément stabilisée au stade du complexe primaire (JOUBERT ef OUDART, 1966), des lésions de tuberculose généralisée ont éłé rencontrées, chez ces animaux, 4 fois sur 10 .

TABLEAU $\mathbb{N}^{\circ} \mathrm{V}$

Nature des lésions observées avec Mycobacterizm tuberculosis dans lss aifférentes espèces animales.

\begin{tabular}{|c|c|c|}
\hline $\begin{array}{l}\text { Ispèce } \\
\text { animale }\end{array}$ & $\begin{array}{l}\text { Neture de la } \\
\text { saisie }\end{array}$ & Lésions observées avec M. tuberculosis \\
\hline Porc & Totale & Poursons, fole, rate, reins \\
\hline$"$ & $\vec{"}$ & Rate \\
\hline$"$ & Partielle & Ganglions bronchiques et rétropharyngiens \\
\hline Zébu. & Totale & Poumons, foie, rate, reins, plèrre et péritoine \\
\hline$"$ & $"$ & $- \pm d$. \\
\hline$\pi$ & $"$ & Foie, rate, reins, intestins \\
\hline$"$ & $"$ & Pournons, fole, rate \\
\hline " & Partielle & $\begin{array}{l}\text { Tête, collier, ganglions rétropheryngiers et } \\
\text { préscapulazres }\end{array}$ \\
\hline " & $"$ & Poumons \\
\hline$"$ & $"$ & Ganglions bronchiques et rétropharyngiens \\
\hline Taurin & $"$ & Tête, poumons \\
\hline$"$ & $"$ & Collier, ganglions préscapulaires \\
\hline$"$ & $"$ & Ganglions bronchiques \\
\hline
\end{tabular}

Quant aux lésions imputables aux mycobactéries atypiques, elles sont indiquées dans le tableau 6. Là encore, ces lésıons ne semblent pas différer de celles occasionnées par $M$. bovis.

\subsubsection{Discussion des résultats.}

Sı le bacille bovin vient largement en tête parmi les souches isolées, on sera peut-être surpris de la fréquence non négligeable avec laquelle est rencontrée $M$. fuberculosis. Ce bacille a été isolé en effet 1 fois sur 4 chez les porcins. En fait, cette fréquence est très compréhensible, si l'on considère le mode de vie de ces animaux vivant en cohabıtation avec l'homme et se nourrissant en partie des résıdus alımentaires laissés par celui-ci. Par contre, la fréquence relative (4,3 p. 100) avec laquelle ce bacille est rencontré chez les bovins peuf paraître, à première vue, surprenante, du fait du mode d'élevage extensif de ces animaux. II faut cependant se souvenir que les peuhls (1), qui conduisent les troupeaux,

(1) Les peuhls sont une race africaıne à vocation essentiellement pastorale. Ils pratiquent la religion musulmane et, très souvent, le nomadisme ou la transhumance. 
sont en fait en contact étrolt avec leurs animaux, marchant chaque jour, pendant des heures, au milieu de ceux-ci.

On conçout alors très bien dans ces conditions qu'un berger contaminé puisse être à l'origine de ces infestations.

Il peut être intéressant de comparer ces résultats avec ceux obtenus à partir des prélèvements humains au cours de la période 1966 à 1968.

Pour 406 cultures posıtives (souches nouvelles isolées), 342 souches ont pu être identifiées avec les résultats suivanis :

- M. fuberculosis : 328 fois, soit 95,9 p. 100

- M. bovis : 10 fois, soit 8,9 p. 100

- M. atypiques : 4 fois, soit 1,9 p. $100(M$. phlci : 1 fols ;

1 fois ;

$M$. aquae III : 1 fois ; scotochromogène :

M. avium : 1 fois).

TABLEAU N $\mathbb{N}^{\circ}$ VI

Nature des lésions observées avec les mycobactérles atypiques dans les différentes espèces animales.

\begin{tabular}{|c|c|c|c|}
\hline $\begin{array}{l}\text { Espèce } \\
\text { animale }\end{array}$ & $\begin{array}{l}\text { Nature de la } \\
\text { saisie }\end{array}$ & $\begin{array}{l}\text { Type de } \\
\text { mycobactéries }\end{array}$ & $\begin{array}{c}\text { Lésıons observées avec les nycobactéries } \\
\text { dites atypiques }\end{array}$ \\
\hline Pore & Totale & $\begin{array}{l}\text { atypique non } \\
\text { identif́iée }\end{array}$ & $\begin{array}{l}\text { Rate, foie, ganglions bronchiques et } \\
\text { rétropharynglens }\end{array}$ \\
\hline$"$ & non précisée & batteyi & Non précisées \\
\hline Zébu & Totale & $"$ & Foie, ganglions bronchlques \\
\hline$"$ & $"$ & $\begin{array}{l}\text { atypique non } \\
\text { identıflée }\end{array}$ & $\begin{array}{l}\text { Fete, reins, ganglions bronchiques et } \\
\text { préscapulaires }\end{array}$ \\
\hline$"$ & $"$ & $"$ & Fole, rate, ganglions préscapulaires \\
\hline " & Partielle & Aquae $I \bar{I}$ & Rate, poumons, genglions bronchiques \\
\hline$"$ & $"$ & Aquae III & Poumons et genglions bronchiques \\
\hline " & $"$ & kansasii & - $1 \mathrm{~d} \cdot-$ \\
\hline " & $"$ & borstelense & - id. - \\
\hline$"$ & $"$ & $\begin{array}{l}\text { atypique non } \\
\text { identifiée }\end{array}$ & $\begin{array}{l}\text { Ganglions rétropharyngiens, précuraux et } \\
\text { préscapulaires }\end{array}$ \\
\hline$"$ & $"$ & $"$ & $\begin{array}{l}\text { Rate, poumons, tête et ganglions } \\
\text { rétropharyngiens }\end{array}$ \\
\hline$"$ & $"$ & $"$ & Collier \\
\hline Taurin & $"$ & $"$ & Poumons et genglions bronchiques \\
\hline
\end{tabular}

- La fréquence relativement réduite avec laquelle le bacille bovin est rencontré chez l'homme semble en contradiction avec certains résultats de nos enquêtes allergologiques récentes (ALBERT et GIDEL, 1969). Celles-CI ont montré en effet la coexistence fréquente, dans certaines régions (régıons sahéliennes en particulıer), des tuberculoses humaine et bovine dans un même village. Maıs il faut souligner que la quasi-totalité des souches qui ont été isolées chez l'homme proviennent de sujets qui ne sont pas originaires de ces régions et n'ont eu, de ce fait, que des contacts très indirects avec les bovins.

Enfin, retenons la relative fréquence de la tuberculose chez les chevaux, 9 cas sur 3.299 animaux inspectés, soit 1 pour 366 alors qu'en France, avant que ne solt mis en œuvre le plan d'éradication de la tuberculose bovine, elle ne dépassait pas la fréquence de 1 p. 100.000 (JOUBERT ef OUDAR, 1966). 


\section{5. - CONCLUSION}

Ces recherches confirment, sinon l'absence totale du farcın, du moins la rareté de cette affection dans les régions dont les animaux étaient originaires et soulıgnent en même temps l'importance de la tuberculose bovine dans ces mêmes régions.

Si $M$. bovis est bien l'agent le plus fréquemment rencontré chez les animaux, $M$. tuberculosis n'est cependant pas exceptionnel. Ce fait, joint à la présence d'un pourcentage non négligeable de $M$. bovis parmi les souches Isolées chez l'homme, ef surtout, aux résultats de nos enquêtes allergologiques, nous a incités à entreprendre une étude bactériologique de la tuberculose humaine et bovine dans les régions sahélıennes prospectées au cours des enquêtes tuberculıniques. Cette étude permettra de préciser la part qui revient à $M$. tuberculosis et à $M$. bovis, à la fois chez l'homme et chez l'animal et d'éclai- rer ainsi l'épidémiologıe de l'affection dans ces régions.

Organisation de Coopération ef de Coordination pour la lutte contre les Grandes Endémies

$$
\begin{aligned}
& \text { (O. C. C. G. E.) - Centre Muraz } \\
& \text { Bobo Dioulasso - Haute-Volta } \\
& \text { (Directeur : Dr J. H. RICOSSÉ) }
\end{aligned}
$$

\section{Remerciements}

Nous exprimons nos vifs remerciements à : - Messieurs les docteurs CHAMBON ef BRES, Directeurs de l'Institut Pasteur de Dakar et Messieurs les docteurs CAUSSE et SARRAT, Chefs de Service à cet Institut, qui ont bien voulu confirmer ou préciser certaines de nos identifications.

- Monsieur N'TON KEITA, Directeur de l'abattoir de Bobo Dioulasso et ses collaborateurs, qui ont accepté de se charger de la récolte des prélèvements.

\section{SUMMARY}

The mycobacteria from animal origin isolated by the Muraz Centre from 1965 to 1968. Isolation and identification methods. Results

The importance of confiscates for tuberculosis to the Bobo-Dioulasso slaughter houses is indicated by the authors. The frequency of these confiscates reached to 9.61 p. 100 for the caltle $(82,961$ inspected animals $)$ and to 2.15 p. 100 for the pigs (16.992 inspected animals) during the 1965 to 1968 included years. The authors indicate the methods employed for the samples, the isolation and the identification of mycobacteria. Finally, the results obtained concernıng the direct examinations, the cultures and the identification of isolated mycobacteria are explained.

Among the 639 samplings received by the laboratory, 298 cultures were positive and 250 strains were identified.

Mycobacterium bovis has been the most frequently found : 89.6 p. 100 of cases.

Mycobocterium tuberculosis has been yet isolated In 5.2 p. 100 of cases and alypic mycobacteria in 5.2 p. 100 of cases. These results indicate the scarcity of farcy and the importance of bovine tuberculasis in the areas of which the animals were native.

The authors conclude by informıng of their intention to undertake a bacteriological study in the breeding areas of sahel where the late fuberculin investigations have indicated lies between human and bovine tuberculosis. There investigations can allowe to specify the types of mycobacteria and to know better the disease epidemiology in these areas.

\section{RESUMEN}

Las micobacterias de origen animal aisladas en el Centro Muraz de 1965 a 1968. Téenicas de aislamiento y de identificación. Resultados

Por de pronto, los autores notan la importancia de los descomisos para tuberculosis en los maladeros de Bobo-Dioulasso. La frecuencia de estos descomisos 


\begin{abstract}
llegó a 9.61 p. 100 para los bovinos (82.961 animales inspectados) y 2,15 p. 100 para los cerdos (16.992 animales inspectados) durante los años 1965 a 1968 incluso. Despues, indican las técnicas que utilizaron para las muestras, el aislamiento y la identificación de las micobacterias. Por fin, exponen los resultados obtenidos concerniendo a los examenes directos, los cultivos y la identificación de las micobacterias aisladas.

Entre las 639 muestras recibidas en el Laboratorio, 298 cultivos fueron positivos y se pudo identificar 250 cepas. El agente más frecuentemente encontrado es Mycobocterium bovis : en 99,6 p. 100 de los casos.

Sın embargo se aisiaron Mycobacterium Juberculosis en 5,2 p. 100 de los casos y micobacterios atipicas tambıen en 5,2 p. 100 de los casos. No se aısló ninguna cepa de Nacardio farcinica. Estos resultados muestran la rareza de la nocardiosis y la importancia de la tuberculosis de los bovinos en las regiones de las cuales los animales eran orıgınarios.

Emprender un estudıo bacterıológico en las regiones sahelıanas de crianza donde encuestas tuberculinicas recientes mostraron que relaciones existirian entre las tuberculasis humana y bovina, tal es el propósito, notado en la conclusıón, que los autores persiguen. Estas investigaciones permitıran precisar los tipos de micobacterias en causa y mejor conocer la epidemiologia de la enfermedad en estas regıones.
\end{abstract}

\title{
BIBLIOGRAPHIE
}

$\operatorname{ALBERT}$ (J. P.), GIDEL (R.) et RETIF (M.). Contribution à l'étude de l'épidémiologie de la tuberculose humaine en Afrique Occidentale. Résultats de 5 enquêtes effectuées au moyen de tests tuberculiniques et par sondage aléatoire dans différentes zones climatiques de Côte d'lvoire et de HauteVolta. A paraitre in «Revue de Tuberculose et Pneurnologie », 1969.

AMSLER (R.). - Interdépendance pathologique de l'homme et de l'animal en face des tuberculoses. Concours médical, 1969, 18, 3831-3843.

BOISVERT (H.). - L'identification des mycobactéries par l'épreuve de réduction des nitrates. Ann. Inst. Pasteur, 1961, 100, 352-357.

BOISVERT (H.). - Identification de Mycobacterium bovis, BCG, et Mycobacterium microli. Ann. Inst. Pasteur, 1966, III, 2, 180-192.

BOGEN (E.). - Amer. Rev. Tub., 1957, 76, 1110. BONICKE (R.). - Zeitsch. F. Hyg., 1958, 145, 263. BUTTIAUX (R.), BEERENS (H.) et TACQUET (A.). - Manuel de techniques bactériologiques. Parss, Edit. Méd. Flammorion, 1963, $505 \mathrm{p}$.

CANETTI (G.), RIST (N.) et GROSSET (J.). Techniques du test de sensibilité de Mycobacterium tuberculosis aux médicaments antibacillaires par la méthode des proportions. Rev. Tub. Pneumol., 1963, 27, 2-3, 263-272.

CANETTI (G.) et GROSSET (J.). - Techniques ef indications des examens bactériologiques en Tuberculose. Saint-Mandé (94), Edit. de la Tourelle, 1968, $193 \mathrm{p}$.

CASTETS (M.), BOISVERT (H.), GRUMBACH (F.), BRUNEL (M.) et RIST (N.). - Les bacilles tuberculeux de type africain. Note préliminaıre. Rev. Tub. Pneumol., 1968, 32, 2, 179-184.

CASTETS (M.), RIST (N.) et BOISVERT (H.). La variété africaine $d u$ bacille tuberculeux humain. Sixièmes journées médicales de Dakar, 1969, C. R. in Méd. Afr. Noire, 16/4, 321-322.

GIDEL (R.), ALBERT (J. P.) et RETIF (M.). Enquête sur la fuberculose bovine au moyen de tests fuberculiniques dans diverses régions d'Afrique Occidentale (Haute-Volta et Côte d'lvoire). Résultals ef considérations générales. Rev. Elev. Méd. Vét. Poys trop., 1966, $22,2$.

JOUBERT (L.) et OUDAR (J.). - Interiransmissibilité et prophylaxie des tuberculoses humaines ef animales. Le problème actuel des mycobactéries afypiques. Gaz. Méd. France, 1966, 3603-3616. 
KONNO (K.). - Science, 1956, 124, 985.

LANGERON (M.), KUBIN (M.), MATEJKA (M.). - Simple Technique for Classification of Mycobacteria. Symposium on isolation and classification of Mycobacteria. Tuberculosis Research Institude, 1964, Prague. Tchécoslovaquie. 2-6 juin 1964.

LAPEYSONNIE (L.) ef CAUSSE (C.). - Notes sur une méthode de coloration rapide du bacille alcoolo-acido-résistant utilisable pour le dépistage de la tuberculose au cours des examens systématiques des collectivités. Rev. Tub. Pneum., 1960, 24, 9-10, 1044-1047.

OLIVIER (H.R.), 一 Traité de biologie appliquée. Tome II. Les diagnostics bactériologiques. Chapitre IV. Mycobacterium fuberculosis ef autres mycobactéries, 1963, par P. J. COLETSOS, 256-297.

PERPEZAT (A.), MARIAT (F.), DESTOMBES (P.) ef THOME (M.). - Importance du farcin chez le zébu du Tchad. Bull. Soc. Poth. Exot., 1963, 56, 3, 375-383.

REGNOULT (M. G.). - La tuberculose animale dans les territoires Ouest-Africains d'expres- sion française. Rev. Poth. Gén. Phys. Clin., 1963, 573, 1093-1115.

SARRAT (H.), TACQUET (A.) et BOIRON (H.).

- Techniques d'étude, valeur des méthodes et résultats des identifications de mycobactéries isolées à Dakar. Sixièmes journées Médicales de Dakar, 1969, C. R. in Méd. Afr. Noire. SARRAT (H.), TACQUET (A.) et CASTETS (M.). - Les mycobactéries atypiques. Etude des souches isolées à Dakar. Sixièmes journées Médicales de Dakar, 1969, C. R. in Méd. Afr. Noire, 16/4, 329-333.

STONEBRINK (B.). - Bull. Off. Int. Epiz., 1952, 37,662 .

TACQUET (A.), TISON (F.) et DEVULDER (B.). - Action des mycobactéries sur les dérivés nitrés. Ann. Inst. Pasteur., 1966, 110 , 2, 252-260.

TISON ( $F$.) et DEVULDER (B.). - Techniques actuelles d'isolement et d'identification des mycobactéries. Path. Biol., 1965, 13, 7-, $458-462$.

VIRTANEN (S.). - A study of nitrate reduction by mycobacteria. Acta Tub. Scandinavia, 1960, supplément 48 . 